\title{
Fatigue assessment of stringer beams using structural health monitoring
}

\author{
Jens Häggström, Thomas Blanksvärd, Peter Collin \\ Luleå University of Technology, Luleå, Sweden
}

Contact: jens.haggstrom@ltu.se

\begin{abstract}
Fatigue assessment of existing bridges is often carried out through simple calculations where the nominal stress range is compared with the fatigue strength based on a number of detail categories specified in codes. Presented in this paper, is the stepwise fatigue assessment through measurements of the 60 year old bridge over Rautasjokk located in northern Sweden. According to the code-based assessment of the stringers, it has already exceeded its lifetime about four times; however no cracks have been identified. By measuring strains the real state of stress was identified, where both nominal stresses and local approaches have been evaluated and compared. Even though the local approach should provide a better accuracy in comparison with the nominal stresses, this approach was only favorable for one out of the three studied locations.
\end{abstract}

Keywords: Assessment, Bridge, Fatigue, Hot-spot, Steel, Stringers, Structural health monitoring. 


\section{Introduction}

Assessment of existing bridges is becoming more essential as the stock and the awareness of sustainability is increasing. The assessment process can be more or less sophisticated, cumbersome and accurate, depending on the asset to be evaluated and the information to be obtained. In general, the best final assessment approach is the cheapest method which shows that the structure have sufficient capacity [1].

The need for assessing a structure is often the consequence of something happening. It can be settlements exceeding what is expected, severe cracking of concrete, initiation of fatigue cracks and changes in traffic volume or loads. It can also be to postpone the exchange of bridges which already has served their theoretical lifespan. The methodology for performing these assessments are somewhat varying, but all with the aim of proving that the capacity is sufficient to withstand the effects for which the structure is being exposed to, for a given period of time with a given safety level. Simplified it can be said that:

$$
R>\mathrm{E}
$$

$R$ represents the capacity and $E$ the Load effect. Both $\mathrm{R}$ and $\mathrm{E}$ consist of stochastic variables, with a given mean value and standard deviation. Most guidelines and codes use characteristic values together with partial coefficients as a simplified approach for adding safety to account for the spread in material properties and loads together with conservative code-based checks.

In several frameworks for assessing bridges a stepwise methodology is suggested, where the initial checking is performed with simplified conservative methods $[2,3,4]$.as shown in Figure 1. The idea is to not do more than what is necessary, meaning that if the bridge is working properly and there are no changes in traffic - there is basically no need to do anything about the bridge. But if something were to happen, which causes doubts regarding the capacity of the bridge, actions needs to be taken.

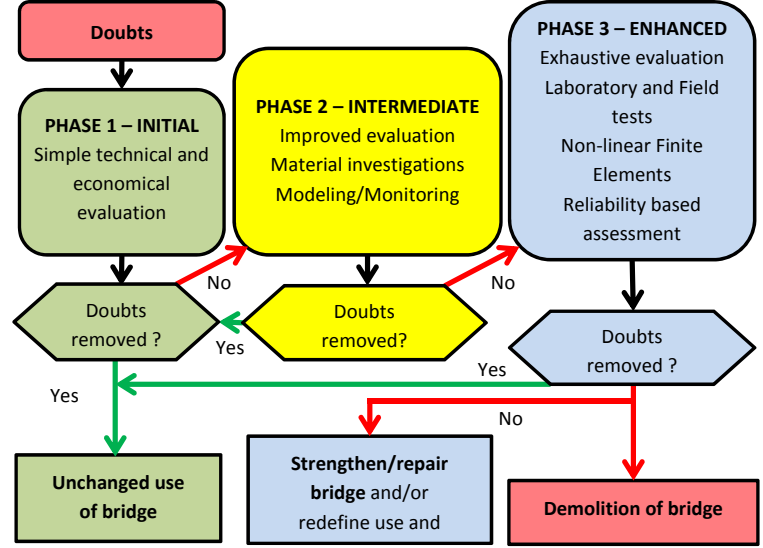

Figure 1 Simplified guidelines for assessment of bridges, (Sustainable bridges, 2007)

The model proposed within the Sustainable bridges suggests three steps of analysis. Initial, Intermediate and Enhanced. The most favourable way to upgrade a bridge is of course to perform administrative upgrading, meaning that the safety level can be ensured though more refined assessment methods - performed in the steps mentioned above.

The aim in this work is to reduce uncertainties and thereby increase $R$ and decrease $E$ as well as reduce the spread of the variance so that the safety can be guaranteed. Structural health monitoring is one possible solution in performing this work, which can be used in order to reduce uncertainties connected to loads and load effects. By performing measurements on live loading for railway bridges one can perform an assessment based on the real state of stress based on the actual loading the bridge is being exposed to. The efforts put in to this, should be weighted to both consequences of failure as well as costs. If the assessment does not provide sufficient capacity suitable measurements must be implemented. The possibilities include strengthening, repair, intensifying inspections, reducing loads or exchanging the structure.

This paper will briefly present the project related to two $33 \mathrm{~m}$ long steel truss railway bridges in northern Sweden, built during the fifties and sixties using the same design. These bridges have been tested in three phases with different focus. Phase 1 was focusing on serviceability limit state while the bridge over Åby (referred to as the Åby Bridge) river still was in service and subjected to 
live loading. For this some 80 sensors were used emphasis mostly on strain gauges even though LVDT's were also used. Phase 2 was more focused on Ultimate limit state, where the old Åby Bridge was exposed to static loading, and eventually loaded to failure. Besides the sensors used in Phase 1, some additional 50 sensors were added, especially LVDTs. Phase 3 was carried out on a different but similar bridge (The bridge over Rautasjokk, which, hereon is referred to as the Rautasjokk bridge) some $500 \mathrm{~km}$ from the Åby bridge. The instrumentation of this bridge was more focused on assessing fatigue through local approaches since this proved insufficient according to the code based assessment performed for the bridge.

\section{Objects}

The studied objects consist of two $33 \mathrm{~m}$ long unballasted steel truss railway bridges. The Åby Bridge was constructed in 1957 and the Rautasjokk bridge 1962, in the technical transition period from riveted to welded bridges. The bridges were therefore partially riveted and partially welded. The rail is supported by wooden cross ties, resting directly on the longitudinal stringers. The stringers span 4,125 meters between the cross beams which are carried by the main truss. In Figure 2 are the two bridges illustrated.

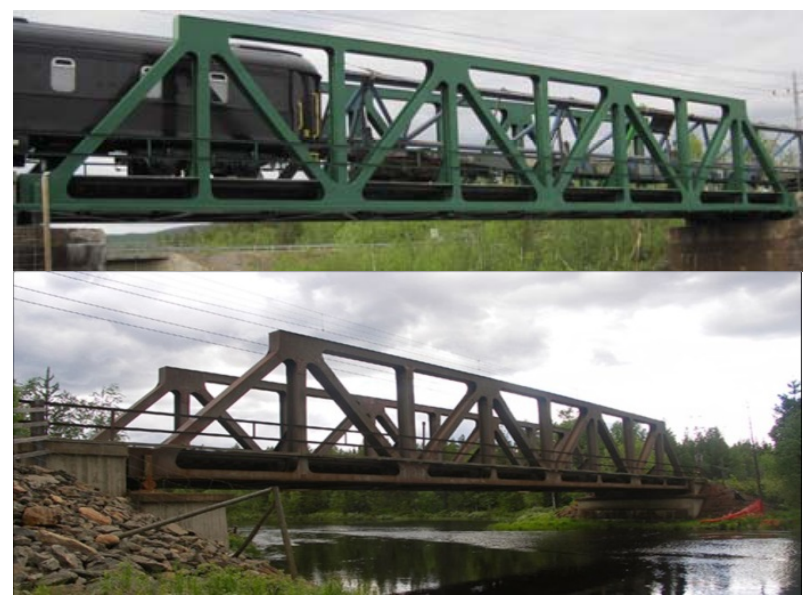

Figure 2 Upper: The Bridge over Rautasjokk, Lower: The Bridge over Åby bridge.

The bridges was designed for a single track with the load according to type F46 which corresponds to 12 axles of 25 tons representing the locomotive and a distributed load of $85 \mathrm{kN} / \mathrm{m}$ representing the wagons [5].

The Åby Bridge was located along the Swedish mainline, the only electrified railway connecting the north and the south of Sweden until it was exchanged 2012in favour for a new ballasted trough bridge.

The Rautasjokk Bridge is located some $20 \mathrm{~km}$ north of Kiruna in northern Sweden along the "Iron ore line" and is still in service. The axle load on this route is some of the highest in Europe due to the mining activities with the current axle load being $300 \mathrm{kN}$ which is about to be increased to $325 \mathrm{kN}$ with the intension of increasing it further to $350 \mathrm{kN}$. Since these loads are frequently occurring extensive work has been carried out in upgrading the bridges along this route, where the Rautasjokk bridge being one of them.

\section{Code based assessment of the Rautasjokk bridge}

\subsection{Ultimate Limit State}

The load carrying capacity of the Rautasjokk bridge [6] was assessed in accordance to the Swedish assessment code [7] for existing railway bridges. The analysis proved that the bridge can withstand the load from $325 \mathrm{kN}$ of axle loading and with some minor strengthening of a riveted connection even $350 \mathrm{kN}$ of axle loading for the given train geometry with regard to Ultimate Limit State, fatigue excluded.

When it comes to fatigue, the analysis is usually performed in several steps similar to what is described in Figure 1 where the next step is taken only if the first prove insufficient.

\subsection{Fatigue Limit State}

In the Swedish code, the initial step is to use the stress-ranges already calculated for the analysis in Ultimate Limit State. By using collective parameters describing the variance in stresscycles, together with fatigue classes based on nominal stress-ranges it is possible get a pass or fail for a given detail with a limited effort. Details with an influence length less than $12 \mathrm{~m}$, such as load-distributing systems are accounted for an 
additional $\times 10$ cycles. This approach does not consider the load-history of bridges, making it unfavourable for bridges like the Rautasjokk bridge since the axle loading has almost been doubled since it was taken in to service.

If the first step proves insufficient, damage accumulation based on the hypothesis of Pamgren-Miner is used [8]. For this analysis, the loading history is required, something which in many cases can be a problem. By running each individual train-set representing the load history it is possible to sum up the fatigue damage which the bridge has been exposed to. It is also possible to calculate the remaining life-span based on estimated future traffic. In this assessment, the engineer is supposed to decide whether it is the number of wheels, bogies or trains which is governing the fatigue damage. For a train loaded with iron-ore pulling 62 wagons with 4 axles each, this will affect the result with the magnitude of $62 \times 4=248$. It is not always simple to decide the number of cycles, which leads to conservative assumptions and an exaggerated safety-level. The method usually provides more favourable results in comparison with the initial check. By refining the fatigue-assessment through damage accumulation, several details for the Rautasjokk bridge passed the check which they didn't in the initial estimation but still several details were found to have insufficient capacity.

One way of reducing the uncertainty in deciding the number of cycles and the collective parameter is through rain flow or reservoir counting [9]. The method is suggested by Eurocode SS-EN 1993-1-9. This is a technique which can be used for both for both simulated responses as well as measured in order to count the number and magnitude of each stress-cycle without having to relate them to the number of axles or bogies. This can be performed by simulating an entire train-passage in a numerical model, but can often be simplified by the use of influence-charts if a linear response is expected.

If studying Figure 3; illustrating the influence lines for the mid of the stringer beam at the end span and the cross-beam at mid-span for the studied bridge, a similar response might be expected for a given train passage. For the loading of an Iron-ore train set with an axle load of $325 \mathrm{kN}$, is the stressvariation plotted in Figure 4. As can be seen, is the total stress-variation greater for the cross-beam, reaching some $74 \mathrm{MPa}$ but this particular detail only receives one of these cycles per passage whereas the rest of the cycles are reaching some $35 \mathrm{MPa}$. For the stringer beam it can be observed that the total stress-variation is smaller compared to the cross-beam but that all cycles have a similar magnitude of some $55 \mathrm{MPa}$. For both details the number of cycles is related to the number of wagons, reaching the peak value as the load from four axles from two different wagons are located in the most unfavourable position.

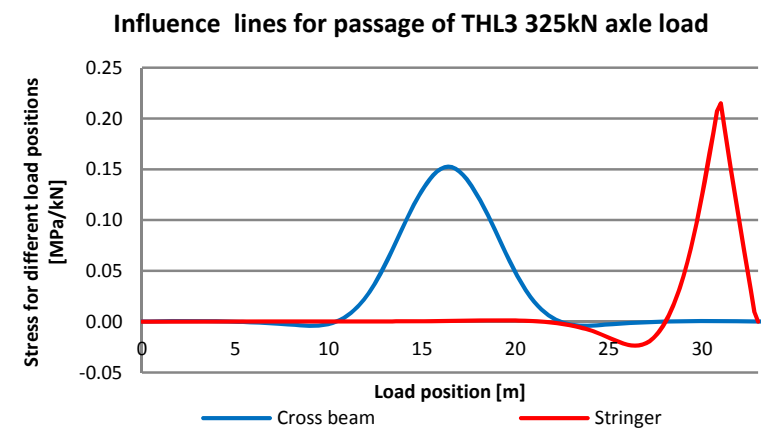

Figure 3 Influence chart for two details on the studied objects.

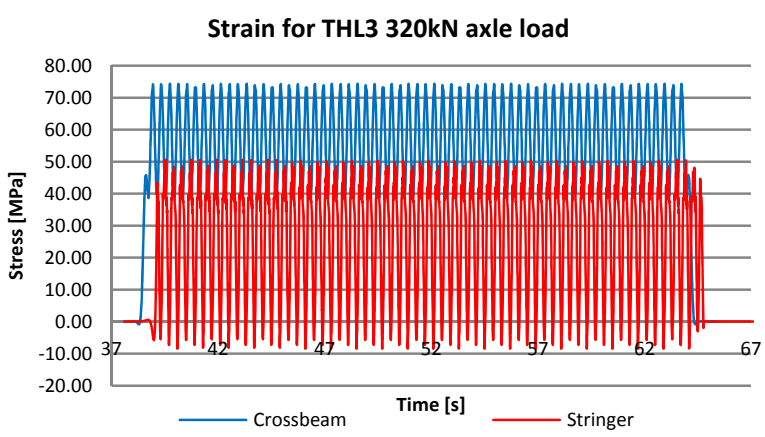

Figure 4 Simulated Stress-variation for a train passage at two different positions based on influence lines.

If disregarding dynamic effects, the train pulling the wagons and the fact that wagons can be unevenly loaded and draw the S-N curve, the result can be observed in Figure 5 . The detail category has been set to $\mathrm{C} 45$ for both the stringer and the cross beam. Train sets consisting of 62 wagons have been assumed and the number of train passages has been set to when the first 
detail reaches an accumulated damage of 1.0, which is reached after some 17600 passages. At this point the stringer has reached an accumulated damage of 1.0 whereas the crossbeam only has consumed $30 \%$ of its capacity with regard to fatigue even though $\Delta \sigma$ was higher for the cross beam and the influence-diagrams had a similar appearance.

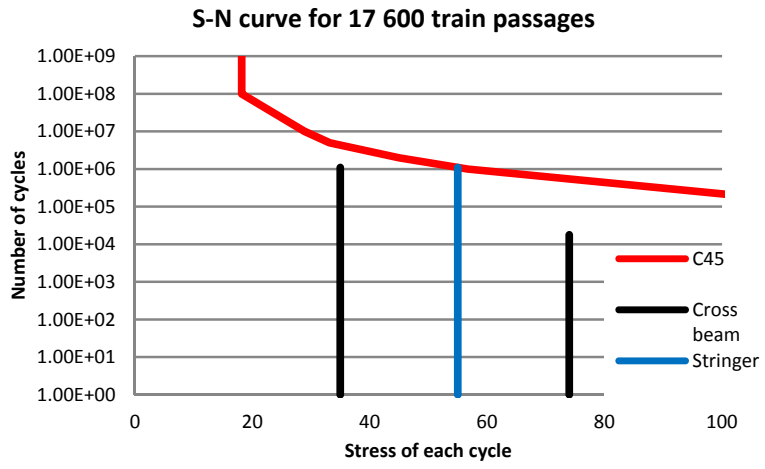

Figure 5 S-N curve for the studied object mentioned above.

This method was method was applied in the fatigue assessment of the Rautasjokk bridge, and it was possible to prove sufficient capacity for all details in the super structure except the connection between the horizontal wind-truss where a steel-plate has been welded to the top flange of the stringer beam, seen in Figure 6 .

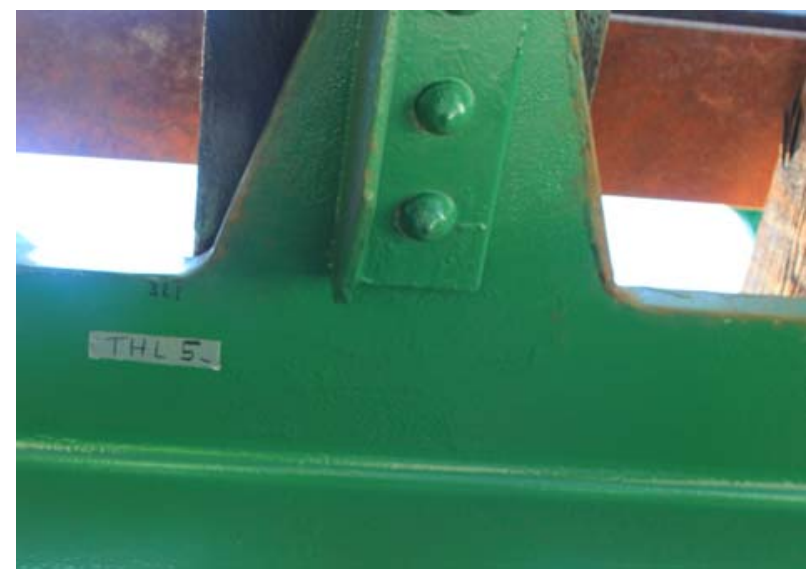

Figure 6 Connection plate welded to the upper flange of the stringer beams, as in point 2.

\section{Details not passing fatigue check}

\subsection{Fatigue sensitive details}

During the time the studied objects were designed, welding with regard to fatigue was a fairly unexplored area. This lead to that fatigue sensitive details were used. One of these details in the studied bridges is the previously mentioned connection plates welded to the upper flange of the stringer beams. The $\mathrm{C}$-class for a detail like this is depending on the relationship between the radius $r$ and width of the plate $I$ as illustrated in Figure 7. The radius for this particular detail was however too small - leaving it in detail category C45 according to Swedish code or C40 according to Eurocode. This resulted in an accumulated damage of 5.2. However no fatigue cracks have been identified, much similar to the Söderströms Bridge in Stockholm [10].

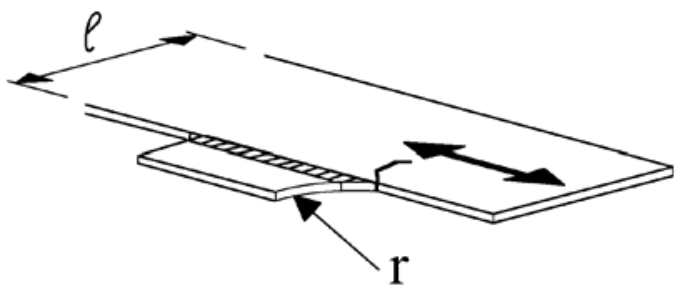

Figure 7 the studied fatigue detail.

The detail is located on the top flange in the middle of each span as can be seen in_Figure 7 . Since the stringers are mainly subjected to bending this will result in compressive stresses in for the critical detail. The assumption of residual stresses close to yielding in tension leads to no improvement of the fatigue resistance without making sure that the tensile residual stresses are removed through post-weld treatment. If looking at the problem from a fracture mechanical point of view, it can be concluded that the crack growth would be much more favorable compared to if the detail would have been in tension.

The thickness of the studied top flange is $24.5 \mathrm{~mm}$ and the connection plate only $10 \mathrm{~mm}$. According to Leander [10] this is likely to be favorable in relationship to the plates having equally thickness.

\subsection{Local approaches}

The reason for the c-class being related to the radius of the connections depends on 3 the level of stress-concentrations related to the particular case.

One way of reducing uncertainties related to generalized C-classes based on nominal stresses 
and cases where an appropriate c-class can't be found is the use of local approaches. One method which can be used is the structural hot-spot stress method. Originally developed for pressure vessels and welded tubular structures in the 1960s [11]. The method is suggested in SS-EN 1993-9. Since structural stress-raisers caused by geometry are taken in to account in the stress calculations the number of S-N curves can be significantly reduced. For the studied detail a c-class of $\mathrm{C}=100$ can be used. It should be mentioned that the fatigue classes cover small imperfections in welds as well as minor misalignments within the tolerances provided in the quality control.

The method is working so that the stress is checked in two or three reference points close to the weld, which then are extrapolated to the weld toe as seen in Figure 8. This leads to that the method is mainly in used for assessing fatigue at the weld toe. Since the method was originally intended to be used together with strain gauges it is possible do perform an assessment based on measurements and numerical simulation as well as measurements. Recommendations for sensor setup and mesh-size are given in $[9,11]$.

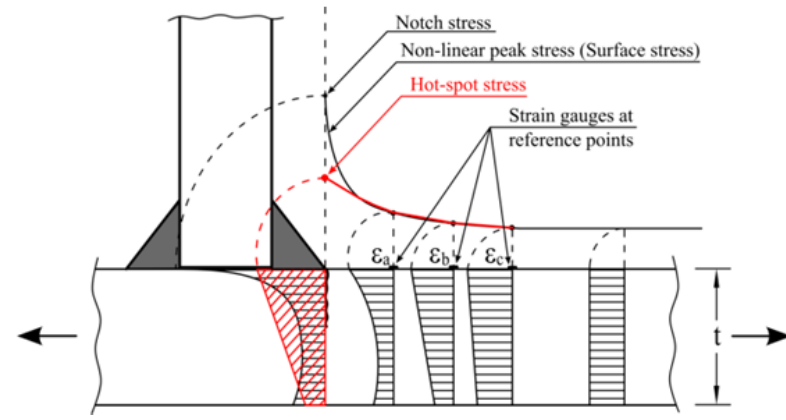

Figure 8 Illustration of extrapolation of hot-spot stresses [11]

\section{Measurements}

The Rautasjokk bridge was in September 2015 monitored with the aim of proving that it had sufficient fatigue capacity. The effect of interaction with the rail and the effect of train speed were also of interest when creating the measurement program but the outcome of this is beyond the scope of this paper. A total of 60 strain gauges was used and monitored the bridge for about two weeks and about 600 trains. Out of those were 130 trains going north loaded with iron ore. The axle load of these trains vary between 280 and $310 \mathrm{kN}$ with and are further analysed, since the trains coming back are significantly lighter since they do not carry any cargo. A trigger was used for the collection of data, so that a high sampling frequency of $400 \mathrm{~Hz}$ could be held without collecting more data than manageable.

The strain gauges used for the hot-spot analysis were coupled strain-gauges so that a fixed distance of $4 \mathrm{~mm}$ between the strain gauges was achieved. These sensors were glued to the steel. For the measurements of nominal strains, normal one-directional strain gauges were used and welded to the steel.

Hot-spot stresses were measured at five different locations and nominal stress was monitored in three of these. The sensor locations can be seen in Figure 9 and Figure 10.

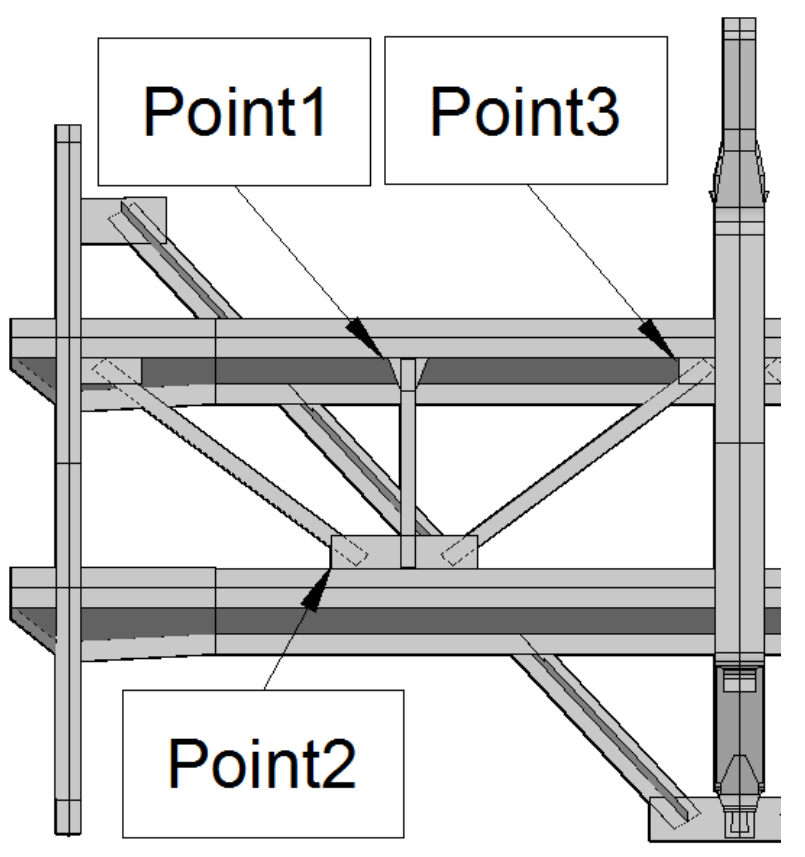

Figure 9 Sensor positions on the stringer beams for measurements on the rautasjokk bridge 


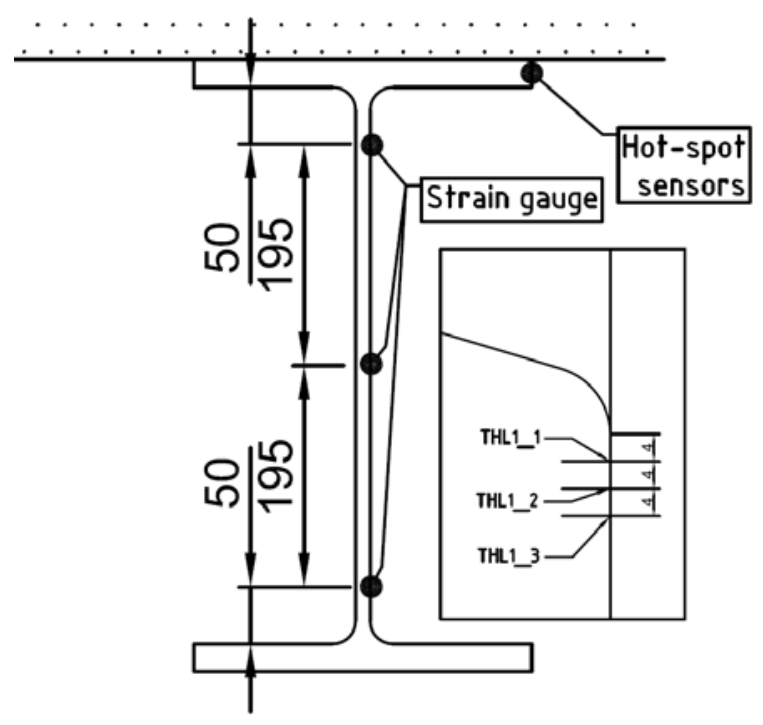

Figure 10 Sensor positions over the cross-section and the configuration of the hot-spot sensors

In Figure 10 are the positions of each sensor illustrated more thoroughly within the section. The hotspot sensors are shown in Figure 11. From these sensors located at $4 \mathrm{~mm}, 8 \mathrm{~mm}$ and $12 \mathrm{~mm}$ from the weld toe are the hot-spot stress extrapolated as:

$\sigma_{h s}=3 * \sigma_{4 m m}-3 * \sigma_{8 m m}+\sigma_{12 m m}$

For the nominal stresses are the strain extrapolated from the strain gauges positioned on the web in an attempt to capture pure nominal strains.

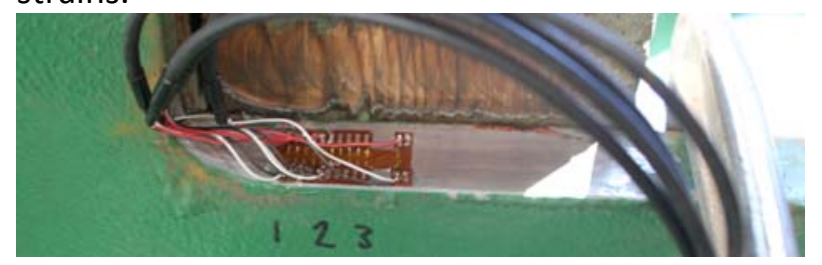

Figure 11 Measurement of hot-spot stresses

\section{Results \& Analysis}

The relationship between nominal stresses and Hot-spot stresses were found to vary a great deal between for the three positions where both nominal and hot-spot stresses were measured. The response from a representative passage can be seen in Figure 12. In the following presented data are strains recalculated through Hooke's law assuming a Young's modulus equal to 210GPa. By the utilizing the Hot-spot method it is possible to increase the fatigue-class for these details with a magnitude of about $100 \%$. This means that the method is favourable if the hot-spot stress is less than twice the magnitude of the nominal stress.
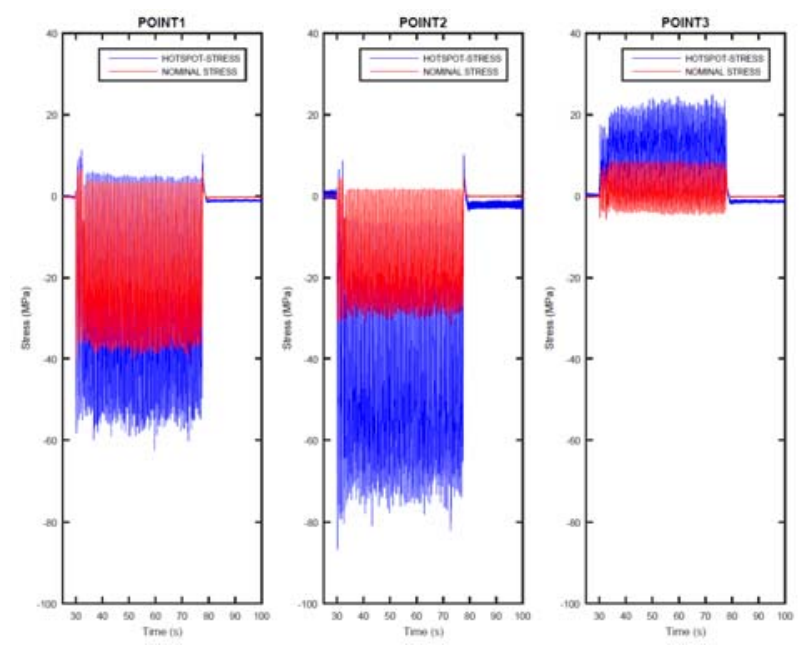

Figure 12 Hot-spot and Nominal stress variation for a passage of an Iron ore train. The points are referring to the positions in Figure 9.

If comparing point 1 and 2 it can be observed that point 1 has a higher nominal stress but that the hot-spot stress is greater for the point 2 . This is likely because of a smoother transition is achieved for this point since there is an inclination of the connected steel plate leading to a less stressconcentrations. For this passage is the hot-spot method favorable for point 1 where while being unfavorable for point $2 \& 3$. The stress at the distinctive points presented together with the calculated hot-spot stress in Figure 4. As can be expected are the stress-variation greater closer to the weld toe.

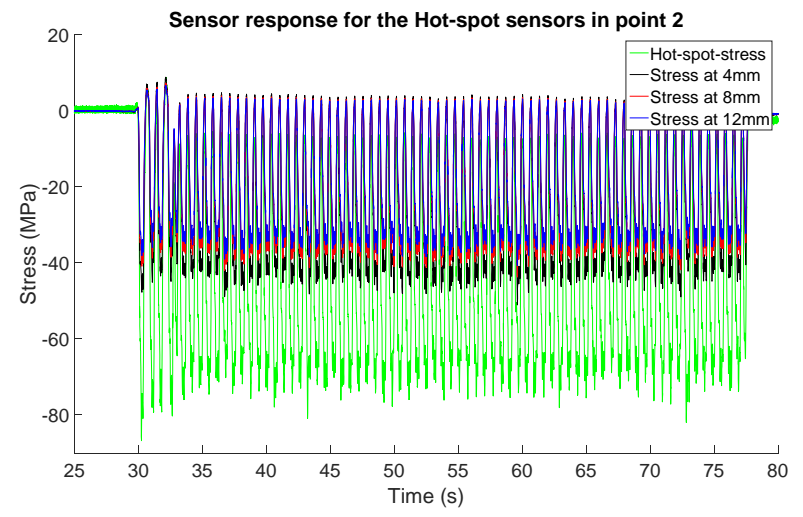

Figure 13 Stress variation at $4 \mathrm{~mm}, 8 \mathrm{~mm}$ and $12 \mathrm{~mm}$ from the weld toe as well as the calculated hot-spot stress. 
The presented data in Figure 13 is only for one single passage. If instead looking at all the loaded iron ore - trains which passed the bridge during the time for measurement we get the histograms presented in Figure 14.

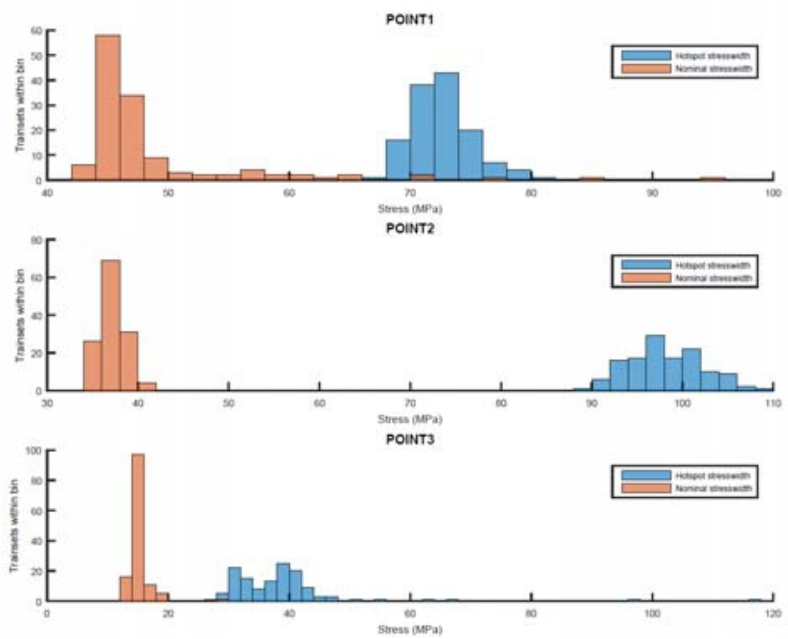

Figure 14 Histograms over stress-widths

By analysing the figure above it is possible so see the effect caused by the geometric stress raisers. For point 1 , is the ratio between the mean hotspot stress and the mean nominal stress 1,49 . For point 2 is the ratio 2,64 and for point 3 it is 2,55. Based on the results are the hot-spot methodology unfavorable to use for point 2 and point 3 . The positioning of the strain-gauges is of course very sensitive, but the results indicate that point 1 is more favorable with regard to stressconcentrations and fatigue. It can also be noted that the nominal stresses are some $10-15 \%$ lower than simulated in Figure 4.

\section{Conclusions \& Future research}

It is inferred that more detailed stress analysis often are required to assess fatigue in critical details in metallic bridges.

For the studied details it can be concluded that the measured stress-levels are smaller than the simulated response despite assuming the stringers continuous over the crossbeams and disregarding dynamic effects. The rail is here believed to act favorable, where it besides distributing forces acts as a structural component together with the stringer beams. The effect the rail has on the stingers will be more thoroughly investigated in future research. Based on the presented data, the hot-spot-method only proved favorable for point 1 , where the shorter length of the connection plate and inclination as seen in Figure 6 and Figure 9 is improving its fatigue resistance.

\section{References}

[1] Tilly G. Iron and steel bridges: condition appraisal and remedial treatment. CIRA C664, London; 2008

[2] Jensen J. Guidelines for load and resistance assessment of existing European railway bridges. Sustainable Bridges. Wroclaw; 2007

[3] Kuhn B. et al. Assessment of Existing Steel Structures: Recommendations for estimation of Remaining Fatigue Life. Background document for the Eurocode. Luxemburg; 2008

[4] Mainline, Assessment methods for elderly rail infrastructure, Universitat Politècnica de Catalunya;2013

[5] Trafikverket. Tåglaster genom tiderna. (Train loads though the ages, in Swedish), version 3.3;2010

[6] Häggström J. Bärighetsberäkning: Bro över södra Rautasjokk KM 1432+883 Kontrollberäkningar för stålöverbyggnad. Luleå; 2014

[7] Trafikverket. Bärighetsberäkning av järnvägsbroar (Capacity calculations of railway bridges - in Swedish). 2013

[8] Miner, M. 1945. Cumulative Damage in Fatigue. Journal of applied mechanics, September 1945.

[9] IIW (International Institute of Welding). Recommendations for fatigue design of welded joints and components. Paris, France, October 2008

[10] Leander J. et al Monitoring and enhanced fatigue evaluation of a steel railway bridge. Engineering structures; 2009

[11] Al-Emrani M, Aygül M. Fatigue design of steel and composite bridges. Chalmers University of technology. Sweden; 2014 